\title{
Association of hip pain with radiographic evidence of hip osteoarthritis: diagnostic test study
}

\author{
Chan Kim, ${ }^{1,2}$ Michael C Nevitt, ${ }^{3}$ Jingbo Niu, ${ }^{1}$ Mary M Clancy, ${ }^{1}$ Nancy E Lane, ${ }^{4}$ Thomas M Link, ${ }^{5}$ \\ Steven Vlad, ${ }^{6}$ Irina Tolstykh, ${ }^{3}$ Pia M. Jungmann, ${ }^{7}$ David T Felson, ${ }^{1,8}$ Ali Guermazi ${ }^{9}$
}

${ }^{1}$ Clinical Epidemiology Research and Training Unit, Boston University School of Medicine, 650 Albany St, Room X200, Boston, MA 02116, USA

${ }^{2}$ Department of Rheumatology, Boston University School of Medicine, Boston, MA, USA ${ }^{3}$ Department of Epidemiology and Biostatistics, University of California, San Francisco, CA, USA ${ }^{4}$ Department of Rheumatology, University of California, Davis, CA, USA

5Department of Rheumatology, University of California, San

Francisco, CA, USA

${ }^{6}$ Department of Rheumatology, Tufts Medical Center, Boston, MA, USA

${ }^{7}$ Department of Diagnostic and Interventional Radiology,

Technische Universität

München, Munich, Germany ${ }^{8}$ NIHR Manchester Biomedical Research Unit, Manchester, UK ${ }^{9}$ Department of Radiology, Boston University School of Medicine, Boston, MA, USA

Correspondence to: C Kim bevochan@bu.edu

Additional material is published online only. To view please visit the journal online (http://dx.doi. org/10.1136/bmj.h5983)

Cite this as: $B M$ J 2015;351:h5983 doi: $10.1136 /$ bmj.h5983

Accepted: 24 October 2015

\section{WHAT IS ALREADY KNOWN ON THIS TOPIC}

Hip osteoarthritis is a major source of morbidity, causing pain, gait abnormalities, and functional impairments

The population of over 60 s has more than doubled in the past 30 years in the US, and the cost of osteoarthritis and its morbidities continues to exponentially increase; in the US the estimated yearly cost of osteoarthritis is about $\$ 185.5 \mathrm{bn}$

A hip radiograph is usually obtained in people with hip pain to diagnosis hip osteoarthritis, and those with pain and radiographic evidence of osteoarthritis are characterized as having hip osteoarthritis

\section{WHAT THIS STUDY ADDS}

In two large epidemiological studies, hip pain was not present in many hips with radiographic evidence of osteoarthritis, and many people with painful hips did not have radiographic evidence of hip osteoarthritis

Most older people highly suspected of having clinical hip osteoarthritis (groin or anterior pain and/or painful internal rotation present) did not have radiographic hip osteoarthritis

Diagnosticians may miss many older people with hip osteoarthritis if they rely on radiographic evidence for diagnosis hip pain at other locations or for painful internal rotation. In the Osteoarthritis Initiative study $(\mathrm{n}=4366)$, only $9.1 \%$ of hips in patients with frequent pain showed radiographic hip osteoarthritis, and $23.8 \%$ of hips with radiographic hip osteoarthritis were frequently painful. The sensitivity of definite radiographic hip osteoarthritis for hip pain localised to the groin was $16.5 \%$, specificity $94.0 \%$, positive predictive value $7.1 \%$, and negative predictive value $97.6 \%$. Results also did not differ much for hip pain at other locations.

\section{WHAT THIS STUDY ADDS}

Hip pain was not present in many hips with radiographic osteoarthritis, and many hips with pain did not show radiographic hip osteoarthritis. Most older participants with a high suspicion for clinical hip osteoarthritis (groin or anterior pain and/or painful internal rotation) did not have radiographic hip osteoarthritis, suggesting that in many cases, hip osteoarthritis might be missed if diagnosticians relied solely on hip radiographs.

FUNDING, COMPETING INTERESTS, DATA SHARING See the full paper on thebmj.com for funding. The authors have no competing interests. Additional data are available from bevochan@bu.edu.

\section{Introduction}

Osteoarthritis of the hip is a major source of morbidity, causing pain, gait abnormalities, and functional impairments. According to the Centers for Disease Control and Prevention, more than 332000 total hip replacements are performed each year in the United States, mostly to treat osteoarthritis. In the urban and suburban community of Framingham, Massachusetts, the age standardized prevalence of radiographic evidence of hip osteoarthritis was 19.6\% (from 2002-05), but the prevalence of symptomatic hip osteoarthritis was only $4.2 \% .^{1}$

Hip pain is often the main symptom of hip osteoarthritis that triggers diagnostic evaluation and treatment. To make the diagnosis in people with hip pain, radiography is usually done, and those with pain and radiographic evidence of osteoarthritis are characterized as having hip osteoarthritis. ${ }^{2}$ Though it is well known that radiographic osteoarthritis is often a late finding in those with knee osteoarthritis and that the concordance between knee pain and radiographic osteoarthritis is poor, less is known about the concordance between hip pain and radiographic hip osteoarthritis ${ }^{34}$; this has been assessed by only a few studies. ${ }^{5-10}$ If people with hip osteoarthritis do not have radiographic evidence of the disease, many may not receive a diagnosis and therefore adequate 
treatment. Both the 2012 American College of Rheumatology and the 2014 Osteoarthritis Research Society guidelines for treatment of hip osteoarthritis recommend a combination of drug and non-pharmacological treatments. For example, they recommend appropriate injections in the correct location (hip joint), which would not be considered if the diagnosis were missed. Furthermore, targeted and specific rehabilitation strategies are recommended but differ depending on the whether the patient has hip pain from osteoarthritis or another source. Guidelines recommend non-steroidal anti-inflammatory drugs for hip osteoarthritis, but there are no definitive treatment guidelines on these for hip bursitis, another common cause of hip pain.

Pain in the hip region is related to many causes, and many people with such pain do not have arthritis. However, the combination of pain in the groin associated with limited internal rotation or pain with internal rotation identifies those with hip arthritis and are components of the classification criteria for osteoarthritis. ${ }^{11}$

We examined the concordance between hip pain and radiographic evidence of osteoarthritis and the diagnostic test performance of the radiograph in those with suspected clinical hip osteoarthritis. We evaluated data from two cohort studies where the evaluation of hip pain and radiographic diagnosis of hip osteoarthritis differed slightly.

\section{Methods}

\section{The Framingham Osteoarthritis Study}

A community based study of osteoarthritis was carried out in Framingham, Massachusetts in 2002-05. Participants were recruited from the Framingham community irrespective of symptoms, and every participant had radiographs. Recruitment has been described previously. ${ }^{12}$ To be included participants had to be ambulatory (use of assistive devices such as canes and walkers was allowed) and have no plans to move away from the area for at least five years. Exclusion criteria were a history of bilateral total knee replacement, rheumatoid arthritis (or other forms of inflammatory arthritis), dementia, terminal cancer, or contraindications to magnetic resonance imaging (MRI). For our study, we included participants aged 50 years and older.

For the assessment of symptoms, participants filled out a questionnaire on the presence and frequency of joint symptoms. Using a homunculus in which each hip joint was depicted as a large circle in the groin, we asked participants whether they had hip pain on most days in that region. We defined those who answered yes as having frequent hip pain. If participants had frequent hip pain, we then asked about the location of the pain: groin, front of leg (anterior), outside the leg (lateral), low back, or buttocks. A standardized hip examination was carried out in those who reported frequent hip pain. They were asked about pain during passive internal rotation and palpation over the greater trochanteric region.

Hip images were obtained from a standing long limb film in the anteroposterior plane. The methods for ascertaining radiographic hip osteoarthritis are described elsewhere. ${ }^{1}$ We defined radiographic hip osteoarthritis as a Kellgren-Lawrence grade of 2 or more (definite joint space narrowing in either superolateral or superomedial sites of the hip joint plus a definite osteophyte). An experienced musculoskeletal radiologist (AG) and a rheumatologist (CK) graded the joint space narrowing and size of osteophytes in accordance with the Osteoarthritis Research Society International (OARSI) atlas. ${ }^{13}$ We have previously published data on the interobserver and intraobserver agreement for radiograph readings. ${ }^{1}$

\section{The Osteoarthritis Initiative}

The Osteoarthritis Initiative is a multicenter longitudinal cohort study of osteoarthritis, which recruited 4796 people aged 45-79 during 2003-05 at four centers: Columbus, $\mathrm{OH}$; Providence, RI; Baltimore, MD; and Pittsburgh, PA. The researchers initially recruited participants if they had or were at risk for knee osteoarthritis. Every participant underwent hip imaging. A description of the recruitment process is available (www.oai.ucsf.edu). As with the Framingham Osteoarthritis Study, inclusion criteria required participants to be ambulatory (use of assistive devices such as canes and walkers was allowed) with no plans to move away from the area for at least three years. People were excluded if they had a history of bilateral total knee replacement, bilateral bone-on-bone knee osteoarthritis, rheumatoid arthritis, contraindications to MRI, or comorbidities that might interfere with the ability to participate in a study for four years.

The ascertainment of hip pain in the Osteoarthritis Initiative differed slightly from that of the Framingham Osteoarthritis Study. In the Osteoarthritis Initiative, participants were shown a visual representation of the hip region, indicating the usual locations of osteoarthritis related pain, ${ }^{514}$ and they were asked whether they had pain, aching, or stiffness in the hip on most days of a month during the past year. ${ }^{15}$ Those who answered yes were considered to have frequent hip pain and asked to indicate on the diagram where the pain occurred: groin, front of leg (anterior), outside of leg (lateral), low back, or buttocks. Each hip was evaluated separately.

In the Osteoarthritis Initiative, participants underwent standard anteroposterior pelvic radiography while weight bearing with their feet internally rotated using a V shaped foot angulation frame. Two musculoskeletal radiologists (TML, PMJ) and a rheumatologist (NEL) assessed the radiographs for individual radiographic features of hip osteoarthritis using the OARSI atlas. ${ }^{13}$ Baseline radiographs of 4761 participants were screened for the presence of any hip osteophytes or joint space narrowing. Those without either finding were categorized as having a negative screen result, and a second reader viewed the radiograph for confirmation. If the second reader confirmed a negative screening result the radiographs were not evaluated further. Two readers independently assessed radiographs with a positive result and scored all radiographic features of 
hip osteoarthritis using the OARSI atlas. At least two of three readers adjudicated disagreements between readers by consensus for the presence of any superolateral or superomedial joint space narrowing, femoral or acetabular osteophytes, cysts, sclerosis, or deformity of the femoral head.

We defined definite femoral and acetabular osteophytes and superolateral and superomedial joint space narrowing as grade 2 or more. Hips were classified as showing definite radiographic hip osteoarthritis based on the presence of any of the following: a modified Croft grade of 2 or more ( $\geq 2$ definite osteophytes; definite joint space narrowing; sclerosis, cysts, or deformity); joint space narrowing plus grade 1 or more femoral osteophytes or grade 2 or more acetabular osteophytes; grade 2 or more femoral osteophytes regardless of other features; and superolateral joint space narrowing grade 2 or more or superomedial joint space narrowing grade 3 or more, regardless of other features. Hips were classified as showing possible radiographic hip osteoarthritis when other individual or combinations of indefinite individual radiographic features were present (for example, grade 1 osteophytes and/or grade 1 joint space narrowing), otherwise they were considered "normal."1617 Test-retest reliability of the reading method was evaluated in a random sample of 189 participants, the radiographs of whom were reread blindly by two readers with adjudication of disagreements. Reliability was generally good for medial and lateral joint space narrowing (weighted $\kappa 0.72$ to 0.77 ); superior and inferior femoral osteophytes (0.70 to 0.81); acetabular osteophytes (0.51 to 0.64 ); cysts, sclerosis, or deformity ( 0.53 to 0.55$)$; the three level summary classification (0.72); and presence or absence of definite radiographic hip osteoarthritis ( $\kappa 0.77)$. Two readers blindly assessed the radiographs of 70 participants with negative results for radiographic hip osteoarthritis for individual radiographic features, with adjudication of disagreements. The specificity of screening for the absence of definite radiographic hip osteoarthritis was $99 \%$, and the estimated sensitivity of screening for definite radiographic hip osteoarthritis was 93\%.

\section{Statistical analysis}

To obtain sensitivity, specificity, and positive and negative predictive values of radiographic hip osteoarthritis for clinical symptoms of hip osteoarthritis for both cohorts, we used SAS for statistical analysis. All of these variables were joint specific and we included both hips for each participant.

To determine the presence of joint symptoms we constructed simple Venn diagrams (see supplementary figures) to show the overlap of frequent hip pain and a positive radiographic finding for hip osteoarthritis because frequent hip pain does not necessarily represent hip osteoarthritis.

To determine the frequency of joint symptoms, we used hip pain localized to the groin or anteriorly as the gold standard for detecting hip osteoarthritis; hip pain in these locations is far more likely to represent true hip joint pain (and with patients with inflammatory arthritis excluded) and therefore to identify those with hip osteoarthritis. In addition, in Framingham we examined participants for pain during internal rotation. If they reported hip pain, we also characterized that as hip joint pain and treated it as the gold standard for detecting hip osteoarthritis. In this instance, we used the radiographs as a diagnostic test and the clinical gold standard method as the diagnosis. Therefore we calculated sensitivity as the percentage of participants with the clinical gold standard of hip osteoarthritis with positive radiographic results, and specificity as the percentage of participants with hips without the clinical gold standard of hip osteoarthritis with negative radiographic results. The positive predictive value is the proportion of hips with radiographic hip osteoarthritis that had a clinical gold standard for hip osteoarthritis. Lastly, we calculated the negative predictive value as the proportion of hips without radiographic hip osteoarthritis that had no clinical gold standard. In addition to using hip pain with anterior thigh or groin pain as the gold standard, we also present diagnostic test findings for frequent hip pain as the gold standard even though hip pain is not a good proxy for hip osteoarthritis.

We excluded replacement hips from analysis. For both cohorts, analysis was done to examine hip pain localized to other regions (lower back, buttocks, or lateral), but these locations had similar poor agreements with positive radiographic findings.

\section{Patient involvement}

No patients were involved in setting the research question or the outcome measures, nor were they involved in developing plans for recruitment, design, or implementation of the study. No patients were asked to advice on interpretation or writing up of results. There are no plans to disseminate the results of the research to study participants or the relevant patient community.

\section{Results}

\section{The Framingham Osteoarthritis Study}

The prevalence of radiographic hip osteoarthritis was $13.6 \%$ (95\% confidence interval $10.7 \%$ to $16.5 \%$ ) for women and $24.7 \%$ (20.6\% to $28.7 \%$ ) for men. ${ }^{1}$ Pain in either hip was reported by $24.7 \%$ (21.2\% to $28.6 \%$ ) of women and $14.7 \%$ (11.4\% to $18.0 \%)$ of men. ${ }^{1}$ For this analysis, radiographs from 1850 hips in 946 participants were evaluated. Table 1 shows the characteristics of the cohort and hip based prevalence of pain by location.

Concordance between frequent hip pain and radiographic evidence of hip osteoarthritis. Radiographs showed a sensitivity of $15.6 \%$ and specificity of $90.9 \%$ for radiographic hip osteoarthritis; the positive predictive value was $20.7 \%$ and negative predictive value $87.6 \%$ (see supplementary figure 1).

For the diagnostic test of radiographs for clinical symptoms of hip osteoarthritis, the sensitivity of radiographic hip osteoarthritis for hip pain localized to the groin was $36.7 \%$, specificity $90.5 \%$, positive predictive value $6.0 \%$, and negative predictive value $98.9 \%$ (table 2 ). The sensitivity of radiographic hip 
osteoarthritis for hip pain localized anteriorly was $24.4 \%$ and specificity $90.4 \%$ (table 2 ). Similarly, the sensitivity of radiographic hip osteoarthritis for hip pain localized to the groin or anteriorly was $27.1 \%$ and specificity $90.6 \%$ (table 2). For participants in the Framingham Osteoarthritis Study, pain with internal rotation during physical examination was also tested. However, despite adding painful internal rotation to the analysis, the agreements of clinical symptoms of hip osteoarthritis and radiographic hip osteoarthritis did not improve. The sensitivity of radiographic hip osteoarthritis for groin or anterior hip pain with painful internal rotation was $33.3 \%$ and specificity $90.3 \%$ (table 2).

\section{The Osteoarthritis Initiative}

Compared with the Framingham Osteoarthritis Study cohort, the Osteoarthritis Initiative cohort had a lower prevalence of radiographic hip osteoarthritis, with definite radiographic hip osteoarthritis present in $7.9 \%$

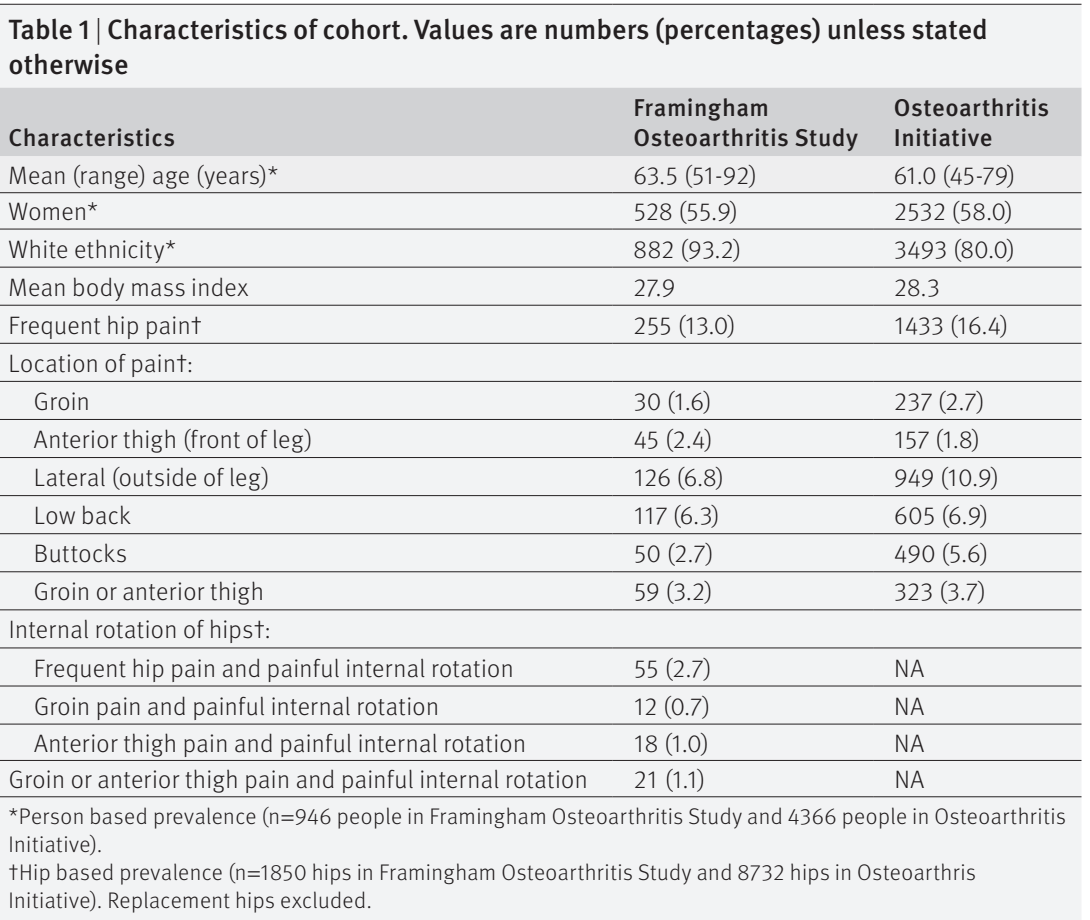

(95\% confidence interval 6.9\% to $9.0 \%$ ) of women and $11.6 \%$ (10.2\% to $13.2 \%$ ) of men. The corresponding prevalence of definite and possible radiographic hip osteoarthritis combined was $20.7 \%$ (19.1\% to $22.3 \%)$ for women and $27.3 \%$ (25.3\% to $29.4 \%$ ) for men. The prevalence of hip pain was similar to that in the Framingham Osteoarthritis Study, with hip pain reported by $27.5 \%$ ( $25.8 \%$ to $29.3 \%$ ) of women and $18.5 \%$ (16.5\% to $20.3 \%)$ of men. Radiographs from 8732 hips in 4366 participants were evaluated.

Concordance between frequent hip pain and radiographic evidence of hip osteoarthritis. The sensitivity of frequent hip pain for radiographic hip osteoarthritis was $9.1 \%$ and specificity $94.3 \%$. The positive predictive value was $23.8 \%$ and negative predictive value $84.1 \%$ (see supplementary figure 2).

For the diagnostic test of radiographs for clinical symptoms of hip osteoarthritis, the sensitivity of definite radiographic hip osteoarthritis for hip pain localized to the groin was $16.5 \%$, specificity $94.0 \%$, positive predictive value $7.1 \%$, and negative predictive value $97.6 \%$ (table 3 ). The sensitivity of radiographic hip osteoarthritis for hip pain localized anteriorly was 15.3\% and specificity 93.9\% (table 3). Similarly, the sensitivity of radiographic hip osteoarthritis for hip pain localized to the groin or anteriorly was $15.8 \%$ and specificity $94.1 \%$ (table 3 ). When we used possible radiographic hip osteoarthritis as the definition for positive radiographic hip osteoarthritis, radiographic hip osteoarthritis was more sensitive (30.0\%) for hip pain localized to the groin or anteriorly, but less specific (81.8\%) (table 4).

\section{Discussion}

Overall, we found poor agreement between frequent hip pain and radiographic osteoarthritis in the ipsilateral hip in participants in both the Framingham Osteoarthritis Study and the Osteoarthritis Initiative. The study methodologies and populations slightly differed between the two cohorts, but the results were similar. Overall, most patients with frequent hip pain did not have radiographic hip osteoarthritis, and most patients with radiographic hip osteoarthritis did not have frequent hip pain. Although frequent hip pain localized to the groin or anterior thigh is commonly thought to

Table 2 | Radiographic hip osteoarthritis (RHOA) and clinical symptoms of hip osteoarthritis in Framingham. RHOA as diagnostic test. Clinical symptoms of hip osteoarthritis as gold standard (symptoms versus none). Values are percentages (number with symptom/total number)

\begin{tabular}{|c|c|c|c|c|}
\hline & Sensitivity & Specificity & $\begin{array}{l}\text { Positive predictive } \\
\text { value }\end{array}$ & $\begin{array}{l}\text { Negative predictive } \\
\text { value }\end{array}$ \\
\hline Clinical symptoms* & $\begin{array}{l}\text { \% of gold standard } \\
\text { hips with RHOA }\end{array}$ & $\begin{array}{l}\text { \% of non-gold standard } \\
\text { hips without RHOA }\end{array}$ & $\begin{array}{l}\% \text { of RHOA hips } \\
\text { with gold standard }\end{array}$ & $\begin{array}{l}\text { \% of non-RHOA hips } \\
\text { without gold standard }\end{array}$ \\
\hline Groin paint & $36.7(11 / 30)$ & $90.5(1647 / 1820)$ & $6.0(11 / 184)$ & $98.9(1647 / 1666)$ \\
\hline Anterior thigh paint & $24.4(11 / 45)$ & $90.4(1632 / 1805)$ & $6.0(11 / 184)$ & $98.0(1632 / 1666)$ \\
\hline Groin or anterior thigh paint & $27.1(16 / 59)$ & $90.6(1623 / 1791)$ & $8.7(16 / 184)$ & $97.4(1623 / 1666)$ \\
\hline Frequent hip pain and painful internal rotation & $18.0(9 / 50)$ & $90.3(1625 / 1800)$ & $4.9(9 / 184)$ & $97.5(1625 / 1666)$ \\
\hline Groin paint and painful internal rotation & $33.3(4 / 12)$ & $90.2(1658 / 1838)$ & $2.2(4 / 184)$ & $99.5(1658 / 1666)$ \\
\hline Anterior thigh paint and painful internal rotation & $27.8(5 / 18)$ & $90.2(1653 / 1832)$ & $2.7(5 / 184)$ & $99.2(1653 / 1666)$ \\
\hline Groin or anterior thigh paint and painful internal rotation & $33.3(7 / 21)$ & $90.3(1652 / 1829)$ & $3.8(7 / 184)$ & $99.2(1652 / 1666)$ \\
\hline
\end{tabular}

*All definitions require pain on most days of a month in past year.

tHips with pain but not in specific location are classified as no pain. Location specific pain includes hips with pain also in other locations. 
Table 3 | Definite radiographic hip osteoarthritis (RHOA) and clinical symptoms of hip osteoarthritis in Osteoarthritis Initiative. RHOA (definite) as diagnostic test. Clinical symptoms of hip osteoarthritis as gold standard (symptoms versus none). Values are percentages (number with pain/total number)

\begin{tabular}{|c|c|c|c|c|}
\hline & Sensitivity & Specificity & $\begin{array}{l}\text { Positive predictive } \\
\text { value }\end{array}$ & $\begin{array}{l}\text { Negative predictive } \\
\text { value }\end{array}$ \\
\hline Pain definition* & $\begin{array}{l}\overline{\%} \text { of gold standard } \\
\text { hips with RHOA }\end{array}$ & $\begin{array}{l}\text { \% of non-gold standard } \\
\text { hips without RHOA }\end{array}$ & $\begin{array}{l}\text { \% of RHOA hips } \\
\text { with gold standard }\end{array}$ & $\begin{array}{l}\% \text { of non-RHOA hips } \\
\text { without gold standard }\end{array}$ \\
\hline Groin paint & $16.5(39 / 237)$ & $94.0(7984 / 8495)$ & $7.1(39 / 550)$ & $97.6(7984 / 8182)$ \\
\hline Anterior thigh paint & $15.3(24 / 157)$ & $93.9(8049 / 8575)$ & $4.4(24 / 550)$ & $98.4(8049 / 8182)$ \\
\hline Groin or anterior thigh paint & $15.8(51 / 323)$ & $94.1(7910 / 8409)$ & $9.3(51 / 550)$ & $96.7(7910 / 8182)$ \\
\hline
\end{tabular}

*All definitions require pain on most days of a month in past year.

tHips with pain but not in specific location are classified as no pain. Location specific pain includes hips with pain also in other locations.

Table 4 | Possible radiographic hip osteoarthritis (RHOA) and clinical symptoms of hip osteoarthritis in Osteoarthritis Initiative. RHOA (possible) as diagnostic test. Clinical symptoms of hip osteoarthritis as gold standard (symptoms versus none). Values are percentages (number with pain/total number)

\begin{tabular}{|c|c|c|c|c|}
\hline & Sensitivity & Specificity & $\begin{array}{l}\text { Positive predictive } \\
\text { value }\end{array}$ & $\begin{array}{l}\text { Negative predictive } \\
\text { value }\end{array}$ \\
\hline Pain definition* & $\begin{array}{l}\text { \% of gold standard } \\
\text { hips with RHOA }\end{array}$ & $\begin{array}{l}\text { \% of non-gold standard } \\
\text { hips without RHOA }\end{array}$ & $\begin{array}{l}\text { \% of RHOA hips } \\
\text { with gold standard }\end{array}$ & $\begin{array}{l}\% \text { of non-RHOA hips } \\
\text { without gold standard }\end{array}$ \\
\hline Anterior thigh paint & $29.9(47 / 157)$ & $81.6(6994 / 8575)$ & $2.9(47 / 1628)$ & $98.5(6994 / 7104)$ \\
\hline Groin or anterior thigh paint & $30(97 / 323)$ & $81.8(6878 / 8409)$ & $6.0(97 / 1628)$ & $96.8(6878 / 7104)$ \\
\hline
\end{tabular}

emanate from hip osteoarthritis, radiographic hip osteoarthritis was not present in most older people with frequent hip pain located primarily in the groin or front of thigh. Also, radiographic hip osteoarthritis was not present in most older people with groin or anterior hip pain with painful internal rotation. We tested the sensitivity of radiographic hip osteoarthritis for clinical symptoms of hip osteoarthritis, but this also tells us the positive predictive value of clinical symptoms of hip osteoarthritis for radiographic hip osteoarthritis. Indirectly, clinical symptoms of hip osteoarthritis were not predictive of radiographic hip osteoarthritis.

Consensus to confirm the diagnosis of hip osteoarthritis is lacking. However, the mention of hip pain triggers a common diagnostic approach: a health professional performs a physical examination to rule out other causes of the pain then orders a pelvic or individual hip radiograph to confirm osteoarthritis. ${ }^{18}$ This assumes that hip pain is predictive of hip osteoarthritis because people with pain in the hip region are likely to have radiographic hip osteoarthritis. Few studies have assessed the concordance between hip pain and radiographic hip osteoarthritis. ${ }^{7814171920}$ One study reported certain radiographic indices associated with higher prevalence of hip pain, and minimal joint space was reported as the best radiographic criterion for hip pain. ${ }^{20}$ Although another study reported a lower prevalence of hip pain ( $7 \%$ in women and $10 \%$ in men) in UK studies compared with our studies, ${ }^{14}$ severe radiographic osteoarthritis was present in $16 \%$ of participants with hip pain compared with $3 \%$ without hip pain. Also, the odds of having hip pain was higher in patients with severe osteoarthritis (17.4, 95\% confidence interval 3 to 102) compared with mild-moderate osteoarthritis (1.4, 0.4 to 4.7$)$.

\section{Limitations of this study}

The largest methodological problem in studying osteoarthritis related pain may be the lack of a validated gold standard for ascertaining that the hip pain is due to osteoarthritis. ${ }^{19}$ Unlike the knee or the ankle, the hip joint has numerous surrounding and adjacent structures that could cause pain in the hip area, such as pelvic disease or referred pain to the hip region. Birrell ${ }^{19}$ studied the construct validity of different approaches to ascertaining hip pain, which included verbal description or visual aid, or both. The combination of both the questionnaires and shaded hip areas on a manikin were superior for association with hip pain indicators (analgesic use, cane use, arthritis consultation) than either questionnaires only or visual aid only. ${ }^{19}$ Our studies combined questionnaires with visual aids to ascertain hip pain. However, we did not assess the severity of hip pain or treatment response to hip osteoarthritis (such as response to non-steroidal anti-inflammatory drugs or corticosteroid injections).

Other methodological problems arise from the use of radiographs to assess hip osteoarthritis. Plain radiographs may lack sensitivity to detect hip osteoarthritis. In knees, small osteophytes may be hidden from overlying boney structures in radiographs, ${ }^{21}$ and this may also be true for small hip joint osteophytes. Similarly, detecting small osteophytes in hip radiographs poses additional challenges because of the more complex variations of the "ball and cup" shape of the hip. Overall, overlying structures can vary substantially between people, and anteroposterior standard radiographs do not adequately visualize large areas, especially in the inferior and posterior hip joint. Weightbearing radiographs of the hip have better sensitivity for hip osteoarthritis than 
non-weightbearing films, including especially weightbearing films acquired using a faux profil oblique view. $^{22-24}$ We did not acquire faux profil views, but these are rarely done as part of the investigations of hip pain in most countries. While weightbearing films may have better sensitivity, the trade-off may be more false positive results and worse specificity (as could be the case for MRI). ${ }^{24}$ Additional films are not necessarily part of common practice for the primary care practitioner. In the Osteoarthritis Initiative, hip radiographs were acquired supine (using a technique similar to that of most radiology suites). In the Framingham Osteoarthritis Study, we acquired hip radiographs as recommended with participants standing, though this is not routinely performed. The diagnostic performances of hip radiographs were not substantially different in these studies.

Studies of knee MRI in Framingham have shown that scanning can detect cartilage damage in those without radiographic knee osteoarthritis, ${ }^{2125}$ and this may also be true of the hip. MRI can also detect other abnormalities not seen on radiographs, such as bone marrow lesions, synovitis, and subtle osteophytes. ${ }^{25}$ No large scale studies have compared hip radiographs with hip MRI, but the few existing studies have shown that hip radiographs are less sensitive in detecting specific features of osteoarthritis than are MRI scans of the hip. ${ }^{26-29}$ In HOAMS (Hip OA MRI scoring systems) studies, hips with a Kellgren-Lawrence grade 0 (no radiographic osteophyte nor joint space narrowing) often showed features of osteoarthritis on MRI ( $25 \%$ of hips with evidence of severe cartilage damage, 33\% with synovitis, and $58 \%$ with labral tears). ${ }^{28}$ For elderly people with chronic hip pain in HOAMS, radiographs were not as sensitive in detecting joint space narrowing as MRI scans. ${ }^{29}$ Though we did not investigate the diagnostic performance of hip MRI, the problems with using MRI to diagnose hip osteoarthritis are likely to be similar to those associated with the knee. In the knee, studies have shown that though MRI is more sensitive than radiography, it is far less specific for abnormalities suggestive of osteoarthritis in most middle aged and older people. ${ }^{25}$

The definition of radiographic hip osteoarthritis has differed in hip osteoarthritis studies, and only a few epidemiology studies have compared the differing definitions of radiographic hip osteoarthritis. ${ }^{230-33}$ The Osteoarthritis Initiative definition derived from the modified Croft definition incorporates more stringent features of hip osteoarthritis (requiring at least joint space narrowing grade $>2$ and/or osteophyte grade $>2$ ), but the Kellgren-Lawrence definition used in the Framingham Osteoarthritis Study is thought to be better associated with osteoarthritis related hip pain and more predictive for total hip replacement. ${ }^{32}$ However, another study ${ }^{17}$ found only minor differences between the Kellgren-Lawrence definition and the Croft definition in predicting total hip replacement, hip pain, and lower extremity disability five years later. Despite differing definitions for radiographic hip osteoarthritis between the Framingham
Osteoarthritis Study and Osteoarthritis Initiative, the results were similar. When a less stringent definition (possible radiographic hip osteoarthritis) was used in the Osteoarthritis Initiative to be more inclusive for detection of hip osteoarthritis, the results did not improve greatly.

The participants recruited for the Osteoarthritis Initiative had or were at risk for knee osteoarthritis, potentially increasing the risk of hip osteoarthritis. Because the positive predictive value and negative predictive value depend on prevalence of the disease, both values in the Osteoarthritis Initiative may be biased. However, the Framingham Osteoarthritis Study was community based and therefore the values are not likely to be biased.

Among limitations of this study are that participants in the Framingham Osteoarthritis Study are mostly white, and the scarcity of members of other race and ethnic groups did not allow for comparisons. This is not true of the Osteoarthritis Initiative, which had larger representations of races. Other limitations include the use of standing long limb films in the Framingham Osteoarthritis Study, which could have led to a slight underestimation of radiographic hip osteoarthritis. However, participants in the Osteoarthritis Initiative had pelvic radiographs, and results were similar to those in the Framingham Osteoarthritis Study. The ascertainment of clinical hip pain differed slightly between the cohorts, but results from the Osteoarthritis Initiative substantiated findings in the Framingham Osteoarthritis Study. Overall, the reproducibility of the discordance between hip pain and radiographic hip osteoarthritis in two different cohorts adds robustness to our findings.

\section{Clinical implications}

We identified and assessed people who most clinicians would characterize as possibly having hip osteoarthritis. In patients aged more than 50 years with groin or anterior hip pain, hip osteoarthritis would be highly suspected, and additional painful internal rotation on physical examination would confirm the clinical diagnosis of hip osteoarthritis for most clinicians. Hip pain and painful internal rotation are features included in the American College of Rheumatology clinical classification criteria for hip osteoarthritis. ${ }^{11}$ Previously, restriction of internal rotation was shown to be the strongest predictor for hip osteoarthritis in a hip range of motion study. ${ }^{7}$ Surprisingly, our inclusion of assessment of reproducible pain with internal rotation did not improve the diagnostic test performance of the radiograph.

In older patients, inadequate recognition of osteoarthritis has consequences. Decreased functional status from osteoarthritis significantly increases morbidity from coronary heart disease, lung disease, diabetes, obesity, falls, frailty, and various other ailments. ${ }^{34}$ Recently, radiographic hip osteoarthritis in older women was found to be associated with an increased risk of mortality from all cause and 
cardiovascular disease among older white women followed for an average of 16 years. ${ }^{35}$ A low number (1 in 10) of patients with osteoarthritis meet adequate physical activity recommendations. Although it has not been shown that increasing physical activity in patients with osteoarthritis leads to lower mortality, better recognition and early treatment of hip osteoarthritis could lead to diminished mortality. The population of people aged over 60 has more than doubled in the past 30 years, and the cost of osteoarthritis and its morbidities will continue to increase exponentially. Currently, \$185.5bn (£122.00bn; €173.00bn) is the estimated yearly cost of osteoarthritis in the United States. ${ }^{36}$ Because many patients with hip pain do not have radiographic hip osteoarthritis, a health professional should continue with the evaluation and treatment of osteoarthritis despite negative radiographic findings.

\section{Strengths of this study}

Our study has important strengths. The Framingham Osteoarthritis Study was community based and recruitment of participants was without reference to joint problems. Although participants in the Osteoarthritis Initiative were originally recruited for a longitudinal study of knee osteoarthritis, the findings in this cohort replicated the similarly poor concordance between hip pain and radiographic hip osteoarthritis seen in the Framingham Osteoarthritis Study. The validity of our findings is suggested by the strong association of prevalence of hip osteoarthritis with age. ${ }^{1}$ We found that women had a higher prevalence of hip pain than men in both the Framingham Osteoarthritis Study and the Osteoarthritis Initiative, and this is consistent with other studies. ${ }^{1437-39}$

\section{Conclusion}

Hip pain is discordant with radiographic hip osteoarthritis. We showed that pain was not present in many hips with evidence of osteoarthritis on radiography, and many painful hips did not show radiographic evidence of hip osteoarthritis. Most older participants highly suspected of having clinical hip osteoarthritis (both groin or anterior pain and/or painful internal rotation) did not have radiographic hip osteoarthritis, suggesting that many older people with hip osteoarthritis might be missed if diagnosticians relied on hip radiographs. Hip osteoarthritis is common in older people with various comorbidities and has major public health cost burdens; therefore health professionals should continue to evaluate and treat patients with hip pain suggestive of osteoarthritis despite negative radiographic findings.

Contributors: CK, MCN, DTF, and AG conceived and designed the study. CK, NEL, TL, PMJ, DTF, and AG acquired the data. CK, JN, SV, MMC, IT, MCN, DTF, and AG analysed and interpreted the data. CK drafted the article, and all authors were responsible for revision of critical important intellectual content. CK is the guarantor. DTF and AG contributed equally to this paper.

Funding: This study was Supported by the National Institutes of Health (NIH AR47785, AG18393, and AR47785), National Institute of Arthritis and Musculoskeletal and Skin Diseases (BAA NHLBI-AR-10-06). The Osteoarthritis Initiative is a public-private partnership comprised of five contracts (N01-AR-2-2258; N01-AR-2-2259; N01-AR-2-2260; N01-AR-2-2261; N01-AR-2-2262) funded by the $\mathrm{NIH}$, a branch of the Department of Health and Human Services, and conducted by the Osteoarthritis Initiative Study Investigators. Private funding partners include Merck Research Laboratories, Novartis Pharmaceuticals, GlaxoSmithKline, and Pfizer. Private sector funding for the Osteoarthritis Initiative is managed by the Foundation for the NIH. This project was also funded by the National Institute of Arthritis and Musculoskeletal and Skin Diseases, NIH, under Contract No HHSN268201000019C. This manuscript was prepared using, in part, an Osteoarthritis Initiative public use dataset and does not necessarily reflect the opinions or views of the Osteoarthritis Initiative investigators, the $\mathrm{NIH}$, or the private funding partners.

Competing interests: All authors have completed the ICMJE uniform disclosure form at www.icmje.org/coi_disclosure.pdf and declare: no support from any organisation for the submitted work no financial relationships with any organisations that might have an interest in the submitted work in the previous three years; no other relationships or activities that could appear to have influenced the submitted work.

Ethical approval: The Framingham study was approved by the institutional review board of Boston University Medical Center. The Osteoarthritis Initiative study was approved by the institutional review board of University of California, San Francisco.

Data sharing: For additional data from the study, contact the corresponding author (bevochan@bu.edu). Additional data from the Osteoarthritis Initiative study are available at https://oai.epi-ucsf.org/ datarelease/.

Transparency: The manuscript's guarantor (CK) affirms that this manuscript is an honest, accurate, and transparent account of the study being reported; that no important aspects of the study have been omitted; and that any discrepancies from the study as planned (and, if relevant, registered) have been explained.

This is an Open Access article distributed in accordance with the Creative Commons Attribution Non Commercial (CC BY-NC 4.0) license which permits others to distribute, remix, adapt, build upon this work non-commercially, and license their derivative works on different terms, provided the original work is properly cited and the use is non-commercial. See: http://creativecommons.org/licenses/ by-nc/4.0/.

Kim C, Linsenmeyer KD, Vlad SC, et al. Prevalence of radiographic and symptomatic hip osteoarthritis in an urban United States community: the Framingham osteoarthritis study. Arthritis Rheumatol 2014;66:3013-7.

2 Nevitt MC. Definition of hip osteoarthritis for epidemiological studies. Ann Rheum Dis 1996;55:652-5.

3 Hannan MT, Felson DT, Pincus T. Analysis of the discordance between radiographic changes and knee pain in osteoarthritis of the knee. Rheumatol 2000;27:1513-7.

4 Bedson J, Croft PR. The discordance between clinical and radiographic knee osteoarthritis: a systematic search and summary of the literature. BMC Musculoskelet Disord 2008:9:116.

5 Birrell F, Croft P, Cooper C, Hosie G, Macfarlane G, Silman A. Health impact of pain in the hip region with and without radiographic evidence of osteoarthritis: a study of new attenders to primary care. The PCR Hip Study Group. Ann Rheum Dis 2000;59:857-63.

6 Arden NK, Nevitt MC, Lane NE, et al. Osteoarthritis and risk of falls, rates of bone loss, and osteoporotic fractures. Study of Osteoporotic Fractures Research Group. Arthritis Rheum 1999:42:1378-85.

Birrell F, Croft P, Cooper C, et al. Predicting radiographic hip osteoarthritis from range of movement. Rheumatology (Oxford) 2001;40:506-12.

8 Birrell F, Afzal C, Nahit E, et al. Predictors of hip joint replacement in new attenders in primary care with hip pain. BrJ Gen Pract 2003;53:26-30.

9 Lane NE, Hochberg MC, Pressman A, Scott JC, Nevitt MC. Recreational physical activity and the risk of osteoarthritis of the hip in elderly women. J Rheumatol 1999;26:849-54.

10 Lane NE, Nevitt MC, Hochberg MC, Hung Y.Y, Palermo L. Progression of radiographic hip osteoarthritis over eight years in a community sample of elderly white women. Arthritis Rheum 2004;50: 1477-86.

11 Altman R, Alarcón G, Appelrouth D, et al. The American College of Rheumatology criteria for the classification and reporting of osteoarthritis of the hip. Arthritis Rheum 1991;34:505-14.

12 Englund M, Guermazi A, Gale D, et al. Incidental meniscal findings on knee MRI in middle-aged and elderly persons. N Engl J Med 2008;359:1108-15.

13 Altman RD, Gold GE. Atlas of individual radiographic features in osteoarthritis, revised. Osteoarthr Cartil 2007;15(Suppl A):A1-56 
14 Birrell F, Lunt M, Macfarlane G, Silman A. Association between pain in the hip region and radiographic changes of osteoarthritis: results from a population-based study. Rheumatology (Oxford) 2005:44:337-41.

15 Lawrence RC, Felson DT, Helmick CG, et al. Estimates of the prevalence of arthritis and other rheumatic conditions in the United States. Part II. Arthritis Rheum 2008;58:26-35.

16 Croft P, Cooper C, Coggon D. Case definition of hip osteoarthritis in epidemiologic studies. J Rheumatol 1994;21:591-2.

17 Arden NK, Lane NE, Parimi N, et al. Defining incident radiographic hip osteoarthritis for epidemiologic studies in women. Arthritis Rheum 2009;60:1052-9.

18 Lane NE. Clinical practice. Osteoarthritis of the hip. N Engl J Med 2007;357:1413-21

19 Birrell F, Lunt M, Macfarlane GJ, Silman AJ. Defining hip pain for population studies. Ann Rheum Dis 2005;64:95-8.

20 Croft P, Cooper C, Wickham C, Coggon D. Defining osteoarthritis of the hip for epidemiologic studies. Am J Epidemiol 1990;132:514-22.

21 Hayashi D, Felson DT, Niu J, et al. Pre-radiographic osteoarthritic changes are highly prevalent in the medial patella and medial posterior femur in older persons: Framingham OA study. Osteoarthr Cartil 2014;22:76-83.

22 Lequesne MG, Laredo JD. The faux profil (oblique view) of the hip in the standing position. Contribution to the evaluation of osteoarthritis of the adult hip. Ann Rheum Dis 1998:57:676-81.

23 Conrozier T, Bochu M, Gratacos J, Piperno M, Mathieu P, Vignon E. Evaluation of the "Lequesne's false profile" of the hip in patients with hip osteoarthritis. Osteoarthr Cartil 1999;7:295-300.

24 Conrozier T, Lequesne MG, Tron AM, Mathieu P, Berdah L, Vignon E. The effects of position on the radiographic joint space in osteoarthritis of the hip Osteoarthr Cartil 1997:5:17-22.

25 Guermazi A, Niu J, Hayashi D, et al. Prevalence of abnormalities in knees detected by MRI in adults without knee osteoarthritis population based observational study (Framingham Osteoarthritis Study). BM/ 2012;345:e5339.

26 Kumar D, Wyatt C, Chiba K, et al. Anatomic correlates of reduced hip extension during walking in individuals with mild-moderate radiographic hip osteoarthritis. J Orthop Res 2015;33:527-34

27 Lee S, Nardo L, Kumar D, et al. Scoring hip osteoarthritis with MR (SHOMRI): a whole joint osteoarthritis evaluation system. J Magn Reson Imaging 2015;41:1549-57.

28 Roemer FW, Hunter DJ, Winterstein A, et al. Hip Osteoarthritis MRI Scoring System (HOAMS): reliability and associations with radiographic and clinical findings. Osteoarthr Cartil 2011;19:946-62.

$29 \mathrm{Xu}$ L. Hayashi D, Guermazi A, et al. The diagnostic performance of radiography for detection of osteoarthritis-associated features compared with MRI in hip joints with chronic pain. Skeletal Radiol 2013;42:1421-8.
30 Gossec L, Jordan JM, Lam M-A, et al. Comparative evaluation of three semi-quantitative radiographic grading techniques for hip osteoarthritis in terms of validity and reproducibility in 1404 radiographs: report of the OARSI-OMERACT Task Force. Osteoarthr Cartil 2009;17:182-7.

31 Jacobsen S, Sonne-Holm S, Søballe K, Gebuhr P. Lund B. Radiographic case definitions and prevalence of osteoarthrosis of the hip: a survey of 4151 subjects in the Osteoarthritis Substudy of the Copenhagen City Heart Study. Acta Orthop Scand 2004;75:713-20.

32 Reijman M, Hazes IM, Pols HA, Bernsen RM, Koes BW, BiermaZeinstra SM. Validity and reliability of three definitions of hip osteoarthritis: cross sectional and longitudinal approach. Ann Rheum Dis 2004;63:1427-33.

33 Reijman M, Hazes JM, Koes BW, Verhagen AP, Bierma-Zeinstra SM. Validity, reliability, and applicability of seven definitions of hip osteoarthritis used in epidemiological studies: a systematic appraisal. Ann Rheum Dis 2004:63:226-32.

34 Misra D, Felson DT, Silliman RA, et al. Knee osteoarthritis and frailty: findings from the Multicenter Osteoarthritis Study and Osteoarthritis Initiative. J Gerontol A Biol Sci Med Sci 2015;70:339-44.

35 Barbour KE, Lui L-Y, Nevitt MC, et al. Hip osteoarthritis and the risk of all-cause and disease-specific mortality in older women: populationbased cohort study. Arthritis Rheumatol 2015;67:1798-805.

36 Kotlarz H, Gunnarsson CL, Fang H, Rizzo JA. Insurer and out-of-pocket costs of osteoarthritis in the US: evidence from national survey data. Arthritis Rheum 2009;60:3546-53

37 Pope DP, Hunt IM, Birrell FN, Silman AJ, Macfarlane GJ. Hip pain onse in relation to cumulative workplace and leisure time mechanical load: a population based case-control study. Ann Rheum Dis 2003:62:322-6.

38 Duncan R, Francis RM, Collerton J, et al. Prevalence of arthritis and joint pain in the oldest old: findings from the Newcastle $85+$ study. Age Ageing 2011;40:752-5.

39 Keenan A-M, Tennant A, Fear J, Emery P, Conaghan PG. Impact of multiple joint problems on daily living tasks in people in the community over age fifty-five. Arthritis Rheum 2006;55:757-64

Supplementary information: Venn diagram showing frequency of hip pain and radiographic hip osteoarthritis in Framingham cohort; Venn diagram showing frequency of hip pain and radiographic hip osteoarthritis in Osteoarthritis Initiative cohort 